\title{
Research on the Establishment of Miao Nationality Embroidery Database in Western Hunan from the Perspective of Digitizing
}

\author{
Longyu Gong ${ }^{1, a}$ \\ ${ }^{1}$ Hunan City University, Hunan, Yiyang, China, 413000
}

Keywords: Establishment, Miao Nationality Embroidery, Database, Western Hunan, Digitizing

\begin{abstract}
As an important intangible cultural heritage of our country, Xiangxi Miao embroidery has unique aesthetic, cultural, economic and social value, and from the perspective of digital protection and inheritance, it is necessary to create a database to explore the effective protection, orderly transmission and benefit promotion of Xiangxi Miao embroidery methods and ways has a very important significance.
\end{abstract}

\section{Introduction}

Chinese embroidery process has a long history, diverse styles, changing methods, the more famous embroidery varieties, including Su embroidery, Guangdong embroidery, Shu embroidery, Beijing embroidery, Gu embroidery, Ou embroidery, Miao embroidery and so on. Which Xiangxi Miao embroidery with its unique practices and artistic style is Chinese important intangible cultural heritage. In recent years, due to the impact of modern industrialization, the transformation of aesthetic taste and folk art rely on the decline of agricultural civilization production methods, resulting in Xiangxi Miao embroidery technology protection and inheritance crisis. Xiangxi Miao embroidery skills face the danger of loss, which requires us to use a variety of methods to protect and inheritance, in order to Xiangxiu this intangible cultural heritage can be passed down from generation to generation. So far, for the traditional means of protection of Miao embroidery, academia has been a lot of discussion, and for the digital history of protection and innovative inheritance related research is still less. Therefore, this article from the digital protection and heritage of the field of view, trying to Xiangxi Miao embroidery database to create the way and achieve ways to achieve system protection and effective heritage.

\section{The Principles and Processes of Miao Embroidery Database Construction}

With the rapid development of non-heritage protection movement, digital technology is also the role of the field is growing, "non-material cultural heritage digital protection" gradually known and used. (1) digital technology for the field of cultural heritage protection, began in the nineties of the twentieth century. Non-digital protection is a system engineering, involving digital acquisition, digital coding, digital storage, digital transmission and other links and steps, they are interlocking, complement each other. Among them, the digital database to create the most cumbersome and complex, is undoubtedly the most important part of the key.

The Principles of Creation of Xiangxi Miao Embroidery Database. Miao embroidery database to create the first to have a comprehensive, comprehensive, including Miao embroidered data categories, content, function several aspects. Miao embroidery database should cover a variety of data categories, including text data, pattern data, color data, cultural data, technical data, process data, etc., constitute a three-dimensional, multi-dimensional data form. In addition, the Miao embroidery database needs to show a comprehensive feature in the data category, including audio data, video data, text data and multimedia data. Finally, the database should have a variety of functions, the Miao embroidery skills and cultural protection, communication, academic research, public entertainment and other uses and functions together, get rid of Miao embroidered database function of a single drawbacks.

The Process of Creation of Xiangxi Miao Embroidery Database Includes Interdisciplinary and Joint Disciplines Talent Collection, Data Collection, Data Collation and Coding and Data 
Improvement. The creation of the Miao embroidery database requires the combination of artistic and technical talents. Basically, non-digital protection is the need for interdisciplinary joint forces to achieve, Miao embroidery database to establish the same. Miao embroidery has a long history, rich cultural connotation, variety, craft techniques are complex, which first need to have in-depth study of Miao embroidery art professionals to check. In addition, the database needs to use the latest digital technology, which also requires the support of digital professional and technical personnel, only through the organic combination of the two and good cooperation in order to achieve good results. Broaden the Miao embroidery data acquisition path, combined with history, text, pictures, field collection, skills and other aspects of data. After the completion of the basic data collection needs to be carefully selected, to keep the essence, the boutique and valuable content left, the data is collated, is conducive to data users search and query. Need to point out that Miao embroidery is also a continuous development of art, the data in the collection process cannot be achieved overnight, so Miao embroidery database should also be a dynamic update, is advancing with the times, the need for immediate new Miao embroidery style to explore the historical data to add and update, so as to achieve the integrity of the database and the times.

\section{Specific Ways and Methods to Create Miao Embroidery Database}

The core work of database creation is the acquisition of Miao embroidery resources. The acquisition of digital resources of Miao embroidery can be divided into new resources collected by field acquisition and field investigation. Specifically, the existing Miao embroidery resources collection is more on the existing, scattered around the various media and literature resources to collect, sort, screen and organize. The specific acquisition path of existing resources can be carried out from the following aspects: first from the research institutions and cultural venues, including institutions of higher learning, museums, art galleries, arts and crafts research clubs, folk museums and various types of libraries to collect the Miao embroidery process and Culture-related literature, through the digital image collection graphic copy scanning and physical paper material collection way. In addition, from the Internet online data collection, on the Miao embroidery introduction, reports, Miao embroidery artists interview records and other information, through direct copy, link, sharing and so on. Collectors can also use the Internet to establish an online platform, the establishment of Miao embroidered art resources collection channels, extensive network communication and Unicom to facilitate the collection of digital data, including Miao embroidery graphic materials, fine patterns, patterns, acupuncture techniques, These network resources are aggregated and collated to transform them into easy-to-identify and easy-to-store data forms sorted storage and network display and dissemination through the work of the technician. In addition, the database should be divided into multiple subdirectories, such as text data, pattern data, pattern data, artist interviews reported data, Miao embroidery finished style data. It should be noted that, in the process of Miao embroidery resources collection cannot ignore the acquisition of academic resources, which is the current non-digital resources in the collection of neglected content. There are various types of academic digital resources, electronic journals, works and design works, and has been published in the traditional academic papers, works, creative works. In the process of collection of flowers, pattern collection, not only to pay attention to those original ecological Miao embroidered clothing on the patterns and patterns, but also those who use the Miao embroidered style of goods, such as certain crafts on the pattern, clothing on the lines and styles to collect, which can be Miao embroidery industrialization, commercialization and mass socialization path to provide a useful reference.

Because of the wide variety of resources, a wide range of forms, so after the basic completion of resource collection, Miao embroidered art experts should be a detailed screening of resources to ensure that the final storage of information authority and artistic value, and then review the data, Heavy, cataloging, editing, etc., making the data become scientific system. From the data format point of view, it is best to choose those who play smooth display, compression capacity is small and the general computer and the media can open the format. For the picture resources, you can unify the use of JPEG format, academic papers and writings in PDF format, reported interview text using DOC format, video files using FLV format, audio files using MP3 format, these files occupy a 
small storage space, compatibility is a better choice. Of course, if there are special documents and special requirements of the file can also be used according to the specific circumstances of other formats.

Xiangxi Miao embroidered the source of resources should also cover the field after the first-hand information, which requires data collection personnel in-depth civil, deep Xiangxi Miao embroidered the birthplace to get. Through the field inspection method to obtain first-hand Miao embroidery information can make up for the lack of information, especially for the Miao embroidery technology techniques to provide a useful supplement. Field research to collect the contents of the information also includes text materials, image data, audio data, video information several categories, but I believe that the field of access to resources is the focus of audio and video information in two areas. Specifically, the field of resources to collect the first collection of scattered in the folk fine Miao embroidered patterns, styles, patterns, such as shooting to collect. Second, it should be Miao embroidery skills, processes, processes, etc. through the implementation of audio and video collection and preservation. For the acquisition of Miao embroidery techniques, the collection of people can visit well-known Miao embroidered artists, especially Miao embroidery representative of the successor, will be some commonly used needle such as flat needle, needle, trocar, needle, mixed needle and so on, the traditional techniques such as editing, around, inlay, stack and so on through the video record. Record commonly used Miao embroidery techniques and technology is because it is commonly used today, but with the passage of time, the descendants of the decline or even disappears and the future is likely to lose. For some special skills and new invention techniques, it should be used slow way to record it. In the recording process, the artist's live demonstrations, oral know-how, graphic description, interpretation of voice and other words together, and strive to make it easy to understand, conducive to heritage. Xiangxi Miao embroidery production process is very complex, and therefore also need to record and save. Collecting people from the Miao embroidered the entire process of weaving, dyeing, sewing, painting tricks, embroidery and other procedures and processes recorded.

After the original data collection, the collectors also need to organize, classify, filter and encode the data, and integrate with the existing data database to create a database that is easy to query and retrieve. These are the necessary steps to preserve the embroidery techniques. After the resource database is built, you can also create a digital pattern database and a computer-aided design system on this basis. Digital pattern database and computer aided design system refers to the pattern of recording, save them in the computer, the establishment of the original pattern database, extract the pattern of the common graphics features and color matching style, the use of artificial intelligence and network technology to extract the data processing, the establishment of computer-aided design database. Digital pattern database is essentially the original Miao embroidery patterns, styles, floral decoration into an independent original unit, auxiliary Miao embroidery design staff to design and innovation, which greatly beneficial to the art of embroidery and innovation is a way worth trying.

\section{The Problems Need to Pay Attention to the Construction of Miao Embroidery Database}

Non-inherited content, is the "surgery" and "Tao" unity, "surgery" is often the external technology, technology, rituals, programs, etc., which is more common content of stability; "Road" is more personality The content of change, is the skills, skills, ideas, beliefs and so on. Xiangxi Miao embroidery as a unique non-material cultural heritage, first reflected in its unique culture, that is, "Road". Therefore, in the process of establishment of Miao embroidery database, especially in the process of digital resource collection, collectors should pay attention to the cultural information of Miao embroidery itself, and excavate the cultural value, such as Miao embroidery history and culture, Miao embroidery pattern, lines, the theme of culture Implication, Miao embroidery produced by the unique geographical culture, and so will really protect the embroidery technology and cultural protection combined.

In addition, Miao embroidery database establishment process should also pay attention to intellectual property issues. In the acquisition process of Miao embroidery data, the collection staff should have a clear understanding of the intellectual property rights of the data. Although the Miao 
embroidery database after the completion of the use of the purpose is to protect and inherit this skill, is a public nature, but the collection of people in the use of books and other information, other database information, especially in the field inspection of Miao embroidery heritage oral, video, The recording of the acquisition process is best to sign the use of intellectual property agreement, so as to avoid future disputes in this regard.

\section{Conclusion}

In the continuous development of new situation of modern digital technology, the establishment of a modern Miao embroidery database to the greatest extent possible to protect and inheritance Miao embroidery skills, production processes, cultural connotation, government and all levels should be joint figures technical staff, Miao embroidered inheritors and art staff to create an effective protection for digital database.

\section{Acknowledgements}

Fund Project: Hunan Province Education Science "Thirteen Five" Planning 2016 General Funding Subject "Research on Ethnic Minority University Art Education and Folk Art Fusion", (subject number: XJK016BMZ001)

\section{References}

[1] Song Junhua, Wang Mingyue. Chinese intangible cultural heritage digital protection of the status quo and problems [J]. Cultural Heritage, 2015 (6)

Journal of Guizhou University of Technology (Natural Science Edition), 2007 (4) (in Chinese with English abstract) [J]. Journal of Guizhou University of Technology (Natural Science Edition), 2007 (4)

[3] Song Junhua. On the intangible cultural heritage digital protection of some thinking [J]. Cultural heritage, 2015 (2) 